\title{
A STRONG CONVERGENCE AND NUMERICAL ILLUSTRATION FOR THE ITERATIVE METHODS TO SOLVE A SPLIT COMMON NULL POINT PROBLEM AND A VARIATIONAL INEQUALITY IN HILBERT SPACES \\ Nguyen Thi Dinh ${ }^{1}$, Pham Thanh Hieu ${ }^{2 *}$ \\ ${ }^{1}$ Hanoi University of Science and Technology \\ ${ }^{2} T N U$ - University of Agriculture and Forestry
}

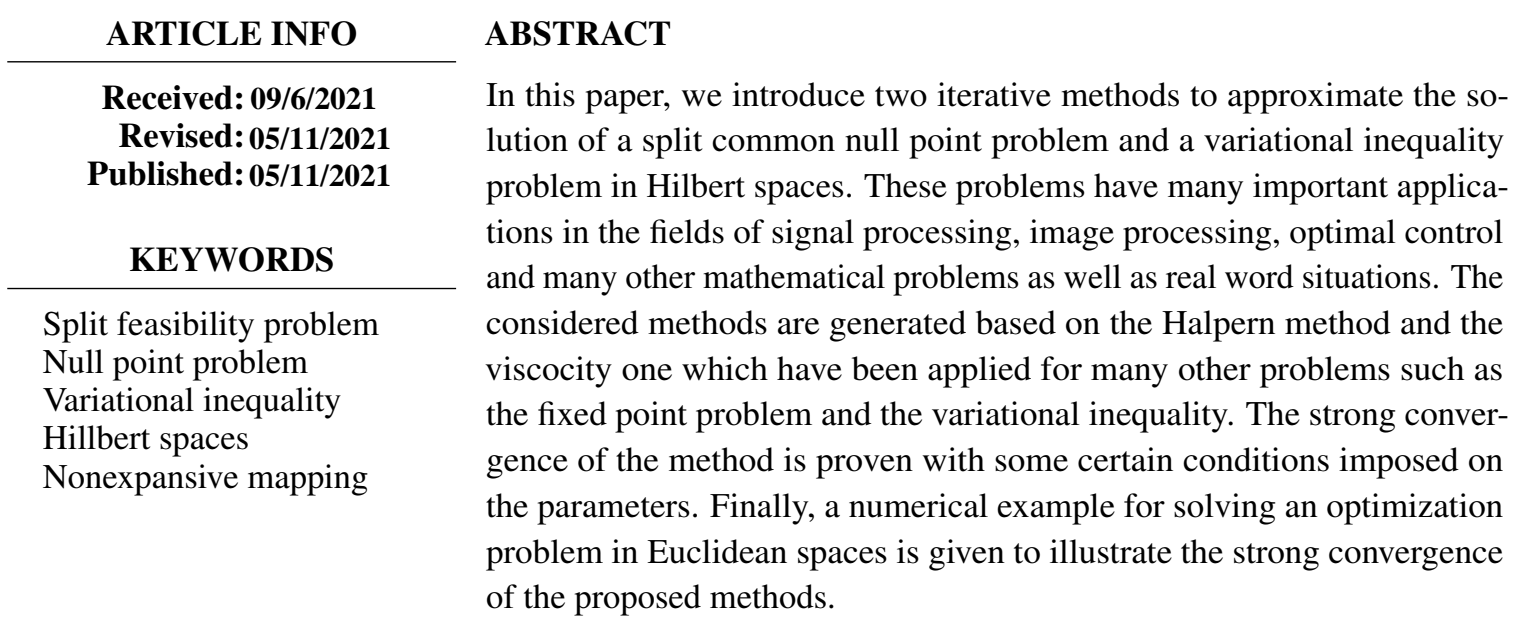

\section{SỰ HộI TỤ MẠNH VÀ VÍ DỤ SỐ CHO PHƯƠNG PHÁP LẶP GIẢI BÀI TOÁN KHÔNG ĐIỂM CHUNG TÁCH VÀ BÀI TOÁN BẤT ĐẲNG THỨC BIẾN PHÂN TRONG KHÔNG GIAN HILBERT}

Nguyễn Thị Dinh ${ }^{1}$, Phạm Thanh Hiếu ${ }^{2 *}$

${ }^{1}$ Trường Đại học Bách khoa Hà Nội

${ }^{2}$ Trường Đại học Nông Lâm - ĐH Thái Nguyên

\section{THÔNG TIN BÀI BÁO}

Ngày nhận bài: 09/6/2021

Ngày hoàn thiện: 05/11/2021

Ngày đăng: 05/11/2021

\section{TỪ KHÓA}

Bài toán chấp nhận tách

Bài toán không điểm

Bất đẳng thức biến phân

Không gian Hilbert

Ánh xạ không giãn

\section{TÓM TẮT}

Trong bài báo này, chúng tôi giới thiệu hai thuật toán lặp để giải bài toán không điểm chung tách và bài toán bất đẳng thức biến phân đơn điệu trong không gian Hilbert. Các bài toán này có nhiều ứng dụng quan trọng trong những lĩnh vực như xử lý tín hiệu, xử lý ảnh, điều khiển tối ưu và nhiều lĩnh vực khác của toán học cũng như trong đời sống. Các phương pháp mà chúng tôi đề xuất dựa trên phương pháp lặp Halpern và phương pháp xấp xỉ mềm đã được áp dụng để giải các bài toán điểm bất động và bất đẳng thức biến phân. Sự hội tụ mạnh của thuật toán đã được chứng minh cùng với một số điều kiện nhất định đặt lên các dãy tham số. Cuối cùng chúng tôi đưa ra một ví dụ số giải bài toán tối ưu trong không gian hữu hạn chiều để minh họa cho sự hội tụ của thuật toán.

DOI: https://doi.org/10.34238/tnu-jst.4619 


\section{Introduction}

Let $C$ and $Q$ be nonempty closed and convex subsets of real Hilbert spaces $H_{1}$ and $H_{2}$, respectively. Let $T: H_{1} \rightarrow H_{2}$ be a bounded linear operator and let $T^{*}: H_{2} \rightarrow H_{1}$ be the adjoint of $T$. The split feasibility problem (SFP) is formulated as follows:

$$
\text { find an element } x^{*} \in S=C \cap T^{-1}(Q) \text {. }
$$

Problem (1) first introduced by Censor and Elfving [1] for modeling inverse problems plays an important role in many disciplines, such as, medical image reconstruction and signal processing. Recently, (1) has been attracted by many mathematicians (see for example, [2] - [4] and references there in).

Problem (1) also consists of so called the convex constrained linear inverse problem as a special case, which is formulated as finding an element $x^{*} \in H_{1}$ such that

$$
x^{*} \in C \text { and } T x^{*}=b \in Q .
$$

In case $C$ is a closed convex subset of a Hilbert space $H$, hence $C$ is the set of null points of the maximal monotone operator $A$, which is defined by $A=\partial i_{C}$, where $i_{C}$ is the indicator function of $C$ and $\partial i_{C}$ is the subdifferential operator of $i_{C}$. So, (1.1) can be deduced from the split common null point problem (SCNPP), which consists of finding a point $x^{*} \in H_{1}$ such that

$$
0 \in A_{1}\left(x^{*}\right) \text { and } 0 \in A_{2}\left(T x^{*}\right),
$$

where $A_{i}: H_{i} \rightarrow 2^{H_{i}}, i=1,2$ are maximal monotone operators.

Let $H_{1}, H_{2}$ be real Hilbert spaces, $A: H_{1} \rightarrow 2^{H_{1}}$ and $B: H_{2} \rightarrow 2^{H_{2}}$ be maximal monotone operators on $H_{1}$, $H_{2}$ respectively. Let $S: H_{1} \rightarrow H_{1}$ be a nonexpansive mapping and $T: H_{1} \rightarrow H_{2}$ be a bounded linear operator. The split common null point problem and a fixed point problem are defined by: find $x^{\dagger} \in H_{1}$ such as

$$
x^{\dagger} \in \Omega:=A^{-1} 0 \bigcap T^{-1}\left(B^{-1} 0\right) \bigcap \operatorname{Fix}(S) .
$$

Now consider the variational inequality problem in Hilbert space. The theory of variational inequality problem (in short VIP) was first considered by Stampacchia [5] in the early 1960s. Since then, it has played the important rule in optimization and nonlinear analysis. To be practice, let $C$ be a nonempty, closed and convex subset of the Hilbert space $H$, and $F: H \longrightarrow H$ be a mapping. Then the VIP is defined as follow:

$$
\text { Find } x^{*} \in C \text { such that }\left\langle F\left(x^{*}\right), x-x^{*}\right\rangle \geq 0 \quad \forall x \in C \text {. }
$$

The (VIP) defined for $C$ and $F$ can be denoted by $\operatorname{VIP}(C, F)$. The applications of the above problems, (SFP)-(SCNP)-(NPF)-(VIP), have been studied and mentioned in many publications, to name a few see [5] [8].

In 2020, Tuyen et al. [4] proved the strong convergence of their hybrid method for solving the split common null point problem and the fixed point problem of a nonexpansive mapping. The medthod is based on the Halpern method [9] and the viscocity one which have been used for fixed point problems and variational inequalities (see $[4,8]$ and references therein). In this paper, based on the method introduced in [4], we present two iterative schemes to solve (2)-(4) for finding a common solution of the split common null point problem and the variational inequality in Hilbert spaces.

The remaining part of this paper is organized as follows: the next section are some notations, definitions and lemmas that will be used for the validity and convergence of the algorithm. The third section is devoted to the proof of our strong convergence result. In Section 4, a numerical example is also discussed to illustrate the convergence of the proposed methods.

\section{Preliminaries}

Let $H$ be a real Hilbert space. In what follows, we write $x^{k} \longrightarrow x$ to indicate that the sequence $\left\{x^{k}\right\}$ converges strongly to $x$ while $x^{k} \rightarrow x$ to indicate that the sequence $\left\{x^{k}\right\}$ converges weakly to $x$.

Definition 2.1. A mapping $F: C \longrightarrow C$ is said to be $L$-Lipschitz continuous if there exists a positive constant $L$ such that

$$
\|F(x)-F(y)\| \leq L\|x-y\| \quad \forall x, y \in C .
$$

If $0<L<1, F$ is said to be contraction mapping. If $L=1, F$ is said to be nonexpansive mapping. 
Definition 2.2. Let $S: C \longrightarrow C$ be a nonexpansive mapping. A point $x \in C$ is said to be a fixed point of $F$ if $F(x)=x$.

Throughout this paper, we denote the set of all fixed points of $F$ by $\operatorname{Fix}(S)$, i.e. $\operatorname{Fix}(S)=\{x \in C \mid S(x)=x\}$.

Definition 2.3. Let $C$ be a set in the Hilbert space $H$. For each $x \in H, P_{C}(x)$ is an element in $C$ such that:

$$
\left\|x-P_{C}(x)\right\| \leq\|x-y\| \quad \forall y \in C .
$$

The mapping $P_{C}: H \longrightarrow C$ is called the metric projection from $H$ onto $C$.

The metric projection $P_{C}$ is a nonexpansive mapping.

For an operator $A: H \rightarrow 2^{H}$, we define its domain, range, and graph as follows:

$$
\begin{aligned}
& D(A)=\{x \in H: A(x) \neq \emptyset\} \\
& R(A)=\cup\{A z: z \in D(A)\}
\end{aligned}
$$

and $G(A)=\{(x, y) \in H \times H: x \in D(A), y \in A(x)\}$, respectively. The inverse $A^{-1}$ of $A$ is defined by $x \in A^{-1}(y)$ if and only if $y \in A(x)$. The operator $A$ is said to be monotone if, for each $x, y \in D(A),\langle u-v, x-y\rangle \geq 0$ for all $u \in A(x)$ and $v \in A(y)$. We denote by $I^{H}$ the identity operator on $H$. A monotone operator $A$ is said to be maximal monotone if there is no proper monotone extension of $A$ or $R\left(I^{H}+\lambda A\right)=H$ for all $\lambda>0$. If $A$ is monotone, then we can define, for each $\lambda>0$, a nonexpansive single-valued mapping $J_{\lambda}^{A}: R\left(I^{H}+\lambda A\right) \rightarrow D(A)$ by

$$
J_{\lambda}^{A}=\left(I^{H}+\lambda A\right)^{-1}
$$

which is called the resolvent of $A$. A monotone operator $A$ is said to satisfy the range condition if $\overline{D(A)} \subset$ $R\left(I^{H}+\lambda A\right)$ for all $\lambda>0$, where $\overline{D(A)}$ denotes the closure of the domain of $A$. For a monotone operator $A$, which satisfies the range condition, we have $A^{-1}(0)=\operatorname{Fix}\left(J_{\lambda}^{A}\right)$ for all $\lambda>0$. If $A$ is a maximal monotone operator, then $A$ satisfies the range condition.

The following lemmas will be needed in what follows for the proof of the main results in this paper.

Lemma 2.1. [4] Let $A: D(A) \subset H \rightarrow 2^{H}$ be a monotone operator. Then, we have the following statements:

(i) for $r \geq s>0$, we have

$$
\left\|x-J_{s}^{A} x\right\| \leq 2\left\|x-J_{r}^{A} x\right\|
$$

for all $x \in R\left(I^{H}+r A\right) \cap R\left(I^{H}+s A\right) ;$

(ii) for all $r>0$ and for all $x, y \in R\left(I^{H}+r A\right)$, we have

$$
\left\langle x-y, J_{r}^{A} x-J_{r}^{A} y\right\rangle \geq\left\|J_{r}^{A} x-J_{r}^{A} y\right\|^{2} ;
$$

(iii) for all $r>0$ and for all $x, y \in R\left(I^{H}+r A\right)$, we have

$$
\left\langle\left(I^{H}-J_{r}^{A}\right) x-\left(I^{H}-J_{r}^{A}\right) y, x-y\right\rangle \geq\left\|\left(I^{H}-J_{r}^{A}\right) x-\left(I^{H}-J_{r}^{A}\right) y\right\|^{2} ;
$$

(iv) if $\Omega=A^{-1}(0) \neq \emptyset$, then, for all $x^{*} \in \Omega$ and for all $x \in R\left(I^{H}+r A\right)$,

$$
\left\|J_{r}^{A} x-x^{*}\right\|^{2} \leq\left\|x-x^{*}\right\|^{2}-\left\|x-J_{r}^{A} x\right\|^{2} .
$$

Lemma 2.2. [6] Let $A: D(A) \subset H \rightarrow 2^{H}$ be a monotone operator. Then, for $\lambda, \mu>0$, and $x \in D(A)$, we have

$$
J_{\lambda}^{A} x=J_{\mu}^{A}\left(\frac{\mu}{\lambda} x+\left(1-\frac{\mu}{\lambda}\right) J_{\lambda}^{A} x\right)
$$

Lemma 2.3. [7] Let $\left\{s_{n}\right\}$ be a sequence of nonnegative numbers, $\left\{\alpha_{n}\right\}$ be a sequence in $(0,1)$ and $\left\{c_{n}\right\}$ be a sequence of real numbers satisfying the conditions:

(i) $s_{n+1} \leq\left(1-\alpha_{n}\right) s_{n}+\alpha_{n} c_{n}$,

(ii) $\sum_{n=0}^{\infty} \alpha_{n}=\infty, \limsup _{n \rightarrow \infty} c_{n} \leq 0$.

Then $\lim _{n \rightarrow \infty} s_{n}=0$.

Lemma 2.4. [10] Let $\left\{x_{n}\right\},\left\{y_{n}\right\}$ be bounded sequences in a Hilbert space $H$ and $\left\{\beta_{n}\right\}$ be a sequence in $(0,1)$ with $\quad 0 \quad<\liminf _{n \rightarrow \infty} \beta_{n} \leq \limsup _{n \rightarrow \infty} \beta_{n}<1$
Let $x_{n+1}=\left(1-\beta_{n}\right) y_{n}+\beta_{n} x_{n}$ for all $n \geq 0$ and $\operatorname{lim\operatorname {lip}_{n\rightarrow \infty }}\left(\left\|y_{n+1}-y_{n}\right\|-\left\|x_{n+1}-x_{n}\right\|\right) \leq 0$.

Then $\lim _{n \rightarrow \infty}\left\|x_{n}-y_{n}\right\|=0$. 


\section{Main Results}

Theorem 3.1. Let $H_{1}$ and $H_{2}$ be two real Hilbert spaces. Let $A: H_{1} \rightarrow 2^{H_{1}}$ and $B: H_{2} \rightarrow 2^{H_{2}}$ be two maximal monotone operators on $H_{1}$ and $H_{2}$, respectively. Let $T: H_{1} \rightarrow H_{2}$ be a bounded linear operator from $H_{1}$ onto $\mathrm{H}_{2}$. Suppose that $\Omega=A^{-1} 0 \cap T^{-1}\left(B^{-1} 0\right) \neq \emptyset$. If the conditions $(C 1)-(C 4)$ as follows are satisfied,

(C1) $\min \left\{\inf _{n}\left\{\gamma_{n}^{A}\right\}, \inf _{n}\left\{\gamma_{n}^{B}\right\}\right\}=r>0$

$$
\lim _{n \rightarrow \infty}\left|\gamma_{n+1}^{A}-\gamma_{n}^{A}\right|=0
$$

(C2) $0<\liminf _{n \rightarrow \infty} \beta_{n} \leq \limsup _{n \rightarrow \infty} \beta_{n}<1$;

(C3) $\sum_{n=1}^{\infty} \alpha_{n}=\infty$ and $\lim _{n \rightarrow \infty} \alpha_{n}=0$;

(C4) $\delta \in\left(0, \frac{2}{\|T\|^{2}}\right)$;

then for any $u, x_{0} \in H_{1}$, the sequence $\left\{x_{n}\right\}$ generated by

$$
\begin{cases}y_{n} & =J_{\gamma_{n}^{A}}^{A} x_{n} \\ z_{n} & =J_{\gamma_{n}^{B}}^{B}\left(T y_{n}\right) \\ t_{n} & =y_{n}+\delta T^{*}\left(z_{n}-T y_{n}\right) \\ x_{n+1} & =\beta_{n} x_{n}+\left(1-\beta_{n}\right)\left[\alpha_{n} u+\left(1-\alpha_{n}\right) t_{n}\right], n \geq 0\end{cases}
$$

where $\left\{\gamma_{n}^{4}\right\},\left\{\gamma_{n}^{B}\right\},\left\{\beta_{n}\right\}$, and $\left\{\alpha_{n}\right\}$, converges strongly to $x^{+}=P_{\Omega}^{H_{1}} u$ as $\rightarrow \infty$.

Proof. Let $p \in \Omega$, from $p \in A^{-1} 0$ and Lemma 2.1 (iv), we have

$$
\left\|y_{n}-p\right\|^{2} \leq\left\|x_{n}-p\right\|^{2}-\left\|x_{n}-J_{\gamma_{n}^{A}}^{A} x_{n}\right\|^{2} .
$$

Since $T p \in B^{-1} 0, B$ is a maximal monotone operator, then $T p=J_{\gamma_{n}^{B}}^{B}(T p)$, using Lemma 2.1 (iv), we get

$$
\left\|z_{n}-T p\right\|^{2} \leq\left\|T y_{n}-T p\right\|^{2}-\left\|T y_{n}-J_{\gamma_{n}^{B}}^{B}\left(T y_{n}\right)\right\|^{2}
$$

Based on Lemma 2.1 (iii), we have the following evaluations

$$
\begin{aligned}
\left\|t_{n}-p\right\|^{2} & =\left\|y_{n}-p\right\|^{2}+\delta^{2}\left\|T^{*}\left(z_{n}-T y_{n}\right)\right\|^{2}+2 \delta\left\langle y_{n}-p, T^{*}\left(z_{n}-T y_{n}\right)\right\rangle \\
& =\left\|y_{n}-p\right\|^{2}+\delta^{2}\left\|T^{*}\left(z_{n}-T y_{n}\right)\right\|^{2}+2 \delta\left\langle T\left(y_{n}-p\right), z_{n}-T y_{n}\right\rangle \\
& =\left\|y_{n}-p\right\|^{2}+\delta^{2}\left\|T^{*}\left(z_{n}-T y_{n}\right)\right\|^{2}-2 \delta\left\langle T y_{n}-T p,-J_{\gamma_{n}^{B}}^{B}\left(T y_{n}\right)+T y_{n}+T p-J_{\gamma_{n}^{B}}^{B}(T p)\right\rangle \\
& \leq\left\|y_{n}-p\right\|^{2}-\delta\left(2-\delta\|T\|^{2}\right)\left\|z_{n}-T y_{n}\right\|^{2} \\
& \leq\left\|x_{n}-p\right\|^{2}-\left\|x_{n}-J_{\gamma_{n}^{A}}^{A} x_{n}\right\|^{2}-\delta\left(2-\delta\|T\|^{2}\right)\left\|z_{n}-T y_{n}\right\|^{2}
\end{aligned}
$$

Let $d_{n}=\alpha_{n} u+\left(1-\alpha_{n}\right) t_{n}$. From (8), we have

$$
\left\|d_{n}-p\right\| \leq \alpha_{n}\|u-p\|+\left(1-\alpha_{n}\right)\left\|t_{n}-p\right\| \leq \alpha_{n}\|u-p\|+\left(1-\alpha_{n}\right)\left\|x_{n}-p\right\| .
$$

Hence, from (9) we can deduce that

$$
\begin{aligned}
\left\|x_{n+1}-p\right\| & \leq \beta_{n}\left\|x_{n}-p\right\|+\left(1-\beta_{n}\right)\left\|d_{n}-p\right\| \\
& \leq \beta_{n}\left\|x_{n}-p\right\|+\left(1-\beta_{n}\right)\left\|d_{n}-p\right\| \\
& \leq\left[1-\alpha_{n}\left(1-\beta_{n}\right)\right]\left\|x_{n}-p\right\|+\alpha_{n}\left(1-\beta_{n}\right)\|u-p\| \\
& \leq \max \left\{\left\|x_{n}-p\right\|,\|u-p\|\right\} \leq \ldots \leq \\
& \leq \max \left\{\left\|x_{0}-p\right\|,\|u-p\|\right\} .
\end{aligned}
$$

Therefor, sequence $\left\{x_{n}\right\}$ is bounded. We also deduce from (6)-(8) that sequences $\left\{y_{n}\right\},\left\{z_{n}\right\}$ và $\left\{t_{n}\right\}$ are bounded. 
Now, we are showing that $\lim _{n \rightarrow \infty}\left\|x_{n+1}-x_{n}\right\|=0$. In deed, from Lemma 2.2, we have

$$
\begin{aligned}
\left\|y_{n+1}-y_{n}\right\| & =\left\|J_{\gamma_{n+1}^{A}}^{A} x_{n+1}-J_{\gamma_{n+1}^{A}}^{A}\left(\frac{\gamma_{n+1}^{A}}{\gamma_{n}^{A}} x_{n}+\left(1-\frac{\gamma_{n+1}^{A}}{\gamma_{n}^{A}}\right) J_{\gamma_{n}^{A}}^{A} x_{n}\right)\right\| \\
& \leq\left\|x_{n+1}-x_{n}\right\|+\frac{\left|\gamma_{n+1}^{A}-\gamma_{n}^{A}\right|}{\gamma_{n}^{A}}\left\|x_{n}-J_{\gamma_{n}^{A}}^{A} x_{n}\right\| \\
& \leq\left\|x_{n+1}-x_{n}\right\|+K_{1}\left|\gamma_{n+1}^{A}-\gamma_{n}^{A}\right|,
\end{aligned}
$$

where $K_{1}=\frac{\sup _{n}\left\{\left\|x_{n}-J_{\gamma_{n}^{A}}^{A} x_{n}\right\|\right\}}{r}<\infty$. Similarly, we also have

$$
\left\|z_{n+1}-z_{n}\right\| \leq T y_{n+1}-T y_{n} \|+K_{2}\left|\gamma_{n+1}^{B}-\gamma_{n}^{B}\right|,
$$

with $K_{2}=\frac{\sup _{n}\left\{\left\|T y_{n}-J_{\gamma_{n}^{B}}^{B}\left(T y_{n}\right)\right\|\right\}}{r}<\infty$. From Lemma 2.1 (iii), we get

$$
\begin{aligned}
\left\|t_{n+1}-t_{n}\right\|^{2}= & \left\|y_{n+1}-y_{n}\right\|^{2}+\delta^{2}\left\|T^{*}\left[\left(z_{n+1}-T y_{n+1}\right)-\left(z_{n}-T y_{n}\right)\right]\right\|^{2} \\
& +2 \delta\left\langle y_{n+1}-y_{n}, T^{*}\left[\left(z_{n+1}-T y_{n+1}\right)-\left(z_{n}-T y_{n}\right)\right]\right\rangle \\
\leq & \left\|y_{n+1}-y_{n}\right\|^{2}+\delta^{2}\|T\|^{2}\left\|\left(z_{n+1}-T y_{n+1}\right)-\left(z_{n}-T y_{n}\right)\right\|^{2} \\
& +2 \delta\left\langle T y_{n+1}-T y_{n},\left(z_{n+1}-T y_{n+1}\right)-\left(z_{n}-T y_{n}\right)\right\rangle \\
\leq & \left\|y_{n+1}-y_{n}\right\|^{2}-\delta\left(2-\delta\|T\|^{2}\right)\left\|\left(z_{n+1}-T y_{n+1}\right)-\left(z_{n}-T y_{n}\right)\right\|^{2} .
\end{aligned}
$$

It follows from $d_{n}=\alpha_{n} u+\left(1-\alpha_{n}\right) t_{n}$ that

$$
\begin{aligned}
\left\|d_{n+1}-d_{n}\right\| & \leq\left|\alpha_{n+1}-\alpha_{n}\right|\|u\|+\left\|\left(1-\alpha_{n+1}\right) t_{n+1}-\left(1-\alpha_{n}\right) t_{n}\right\| \\
& \leq\left|\alpha_{n+1}-\alpha_{n}\right|\|u\|+\left|\alpha_{n+1}-\alpha_{n}\right|\left\|t_{n+1}\right\|+\left(1-\alpha_{n}\right)\left\|t_{n+1}-t_{n}\right\| \\
& \leq\left\|t_{n+1}-t_{n}\right\|+K_{3}\left|\alpha_{n+1}-\alpha_{n}\right|
\end{aligned}
$$

where $K_{3}=\|u\|+\sup _{n}\left\{\left\|t_{n}\right\|\right\}<\infty$. From (10)-(12) và (13), we obtain

$$
\left\|d_{n+1}-d_{n}\right\| \leq\left\|x_{n+1}-x_{n}\right\|+K_{1}\left|\gamma_{n+1}^{A}-\gamma_{n}^{A}\right|+K_{3}\left|\alpha_{n+1}-\alpha_{n}\right| .
$$

Then, from the conditions (C1) and (C3), we have

$$
\lim _{n \rightarrow \infty} \sup \left(\left\|d_{n+1}-d_{n}\right\|-\left\|x_{n+1}-x_{n}\right\|\right) \leq 0 .
$$

So, it follows from Lemma 2.4 that

$$
\lim _{n \rightarrow \infty}\left\|x_{n}-d_{n}\right\|=0
$$

Hence, we have $\left\|x_{n+1}-d_{n}\right\|=\beta_{n}\left\|x_{n}-d_{n}\right\| \rightarrow 0$, which together with (14) yields that

$$
\lim _{n \rightarrow \infty}\left\|x_{n+1}-x_{n}\right\|=0
$$

Next, we prove that the set of weak cluster points of the sequence $\left\{x_{n}\right\}$ is contained in $\Omega$. Indeed, we denote $\omega\left(x_{n}\right)$ the set of weak cluster points of the sequence $\left\{x_{n}\right\}$ and suppose that $x^{*}$ is an arbitrarily in $\omega\left(x_{n}\right)$. Then there is a subsequence $\left\{x_{n_{k}}\right\}$ of $\left\{x_{n}\right\}$ such that $x_{n_{k}} \rightarrow x^{*}$. From the convexity of the function $\|\cdot\|^{2}$ on $H_{1}$ and (8), we deduce that

$$
\begin{aligned}
\left\|x_{n+1}-p\right\|^{2} & \leq \beta_{n}\left\|x_{n}-p\right\|^{2}+\left(1-\beta_{n}\right)\left\|\alpha_{n} u+\left(1-\alpha_{n}\right) t_{n}-p\right\|^{2} \\
& \leq \beta_{n}\left\|x_{n}-p\right\|^{2}+\left(1-\beta_{n}\right)\left[\alpha_{n}\|u-p\|^{2}+\left(1-\alpha_{n}\right)\left\|t_{n}-p\right\|^{2}\right] \\
& \leq\left\|x_{n}-p\right\|^{2}+\alpha_{n}\|u-p\|^{2}-\left\|x_{n}-J_{\gamma_{n}^{A}}^{A} x_{n}\right\|^{2}-\delta\left(2-\delta\|T\|^{2}\right)\left\|z_{n}-T y_{n}\right\|^{2} .
\end{aligned}
$$

Thus, we gain

$$
\begin{aligned}
\left\|x_{n}-J_{\gamma_{n}^{A}}^{A} x_{n}\right\|^{2}+\delta\left(2-\delta\|T\|^{2}\right)\left\|z_{n}-T y_{n}\right\|^{2} & \leq\left(\left\|x_{n}-p\right\|^{2}-\left\|x_{n+1}-p\right\|^{2}\right)+\alpha_{n}\|u-p\|^{2} \\
& \leq\left(\left\|x_{n}-p\right\|-\left\|x_{n+1}-p\right\|\right)\left\|x_{n+1}-x_{n}\right\|+\alpha_{n}\|u-p\|^{2} .
\end{aligned}
$$


It follows from $\left\|x_{n+1}-x_{n}\right\| \rightarrow 0$ and the conditions (C3)-(C4) that

$$
\lim _{n \rightarrow \infty}\left\|x_{n}-J_{\gamma_{n}^{A}}^{A} x_{n}\right\|^{2}=\lim _{n \rightarrow \infty}\left\|z_{n}-T y_{n}\right\|^{2}=0
$$

So, we have

$$
\lim _{n \rightarrow \infty}\left\|x_{n}-J_{\gamma_{n}^{A}}^{A} x_{n}\right\|=\lim _{n \rightarrow \infty}\left\|J_{\gamma_{n}^{B}}^{B}\left(T y_{n}\right)-T y_{n}\right\|=\lim _{n \rightarrow \infty}\left\|t_{n}-y_{n}\right\|=0 .
$$

From Lemma 2.1 (i), we get $\lim _{n \rightarrow \infty}\left\|x_{n}-J_{r}^{A} x_{n}\right\|=\lim _{n \rightarrow \infty}\left\|J_{r}^{B}\left(T y_{n}\right)-T y_{n}\right\|=0$.

Since $x_{n_{k}} \rightarrow x^{*}$, và $\lim _{n \rightarrow \infty}\left\|x_{n}-y_{n}\right\|=0$, one has $y_{n} \rightarrow x^{*}$. Because $T$ is a bounded linear operator, $T y_{n} \rightarrow T x^{*}$. By the use of Lemma 2.4, we obtain $x^{*} \in A^{-1} 0$, and $T x^{*} \in B^{-1} 0$, so $x^{*} \in A^{-1} 0 \cap T^{-1}\left(B^{-1} 0\right)$. Consequently, $\omega\left(x_{n}\right) \subseteq \Omega$.

Finally, we show $x_{n} \rightarrow x^{+}=P_{\Omega}^{H_{1}} u$. By putting $x^{+}=P_{\Omega}^{H_{1}} u$, from (7) we get that

$$
\begin{aligned}
\left\|x_{n+1}-x^{+}\right\|= & \beta_{n}\left\langle x_{n}-x^{+}, x_{n+1}-x^{+}\right\rangle+\left(1-\beta_{n}\right)\left\langle\alpha_{n} u+\left(1-\alpha_{n}\right) t_{n}-x^{+}, x_{n+1}-x^{+}\right\rangle \\
\leq & \beta_{n} \frac{\left\|x_{n}-x^{+}\right\|^{2}+\left\|x_{n+1}-x^{+}\right\|^{2}}{2}+\left(1-\beta_{n}\right)\left(1-\alpha_{n}\right) \frac{\left\|t_{n}-x^{+}\right\|^{2}+\left\|x_{n+1}-x^{+}\right\|^{2}}{2} \\
& +\alpha_{n}\left(1-\beta_{n}\right)\left\langle u-x^{+}, x_{n+1}-x^{+}\right\rangle \\
\leq & \beta_{n} \frac{\left\|x_{n}-x^{+}\right\|^{2}+\left\|x_{n+1}-x^{+}\right\|^{2}}{2}+\left(1-\beta_{n}\right)\left(1-\alpha_{n}\right) \frac{\left\|x_{n}-x^{+}\right\|^{2}+\left\|x_{n+1}-x^{+}\right\|^{2}}{2} \\
& +\alpha_{n}\left(1-\beta_{n}\right)\left\langle u-x^{+}, x_{n+1}-x^{+}\right\rangle .
\end{aligned}
$$

Thus,

$$
\left[1+\alpha_{n}\left(1-\beta_{n}\right)\right]\left\|x_{n+1}-x^{+}\right\|^{2} \leq\left[1-\alpha_{n}\left(1-\beta_{n}\right)\right]\left\|x_{n}-x^{+}\right\|^{2}+2\left(1-\beta_{n}\right) \alpha_{n}\left\langle u-x^{+}, x_{n+1}-x^{+}\right\rangle .
$$

The last inequalities imply that

$$
\left\|x_{n+1}-x^{+}\right\|^{2} \leq\left[1-\alpha_{n}\left(1-\beta_{n}\right)\right]\left\|x_{n}-x^{+}\right\|^{2}+\left(1-\beta_{n}\right) \alpha_{n} \frac{2}{1+\left(1-\beta_{n}\right) \alpha_{n}}\left\langle u-x^{+}, x_{n+1}-x^{+}\right\rangle .
$$

Let $s_{n}=\left\|x_{n}-x^{+}\right\|^{2}$ and $c_{n}=\frac{2}{1+\alpha_{n}\left(1-\beta_{n}\right)}\left\langle u-x^{+}, x_{n+1}-x^{+}\right\rangle$. Then the inequality (17) can be rewritten in the following form

$$
s_{n+1} \leq\left[1-\alpha_{n}\left(1-\beta_{n}\right)\right] s_{n}+\alpha_{n}\left(1-\beta_{n}\right) c_{n} .
$$

Now, we will show that $\limsup _{n \rightarrow \infty} c_{n} \leq 0$. Indeed, suppose that $\left\{x_{n_{k}}\right\}$ is a subsequence of $\left\{x_{n}\right\}$ such that

$$
\lim \sup _{n \rightarrow \infty}\left\langle u-x^{+}, x_{n}-x^{+}\right\rangle=\lim _{k \rightarrow \infty}\left\langle u-x^{+}, x_{n_{k}}-x^{+}\right\rangle .
$$

Since $\left\{x_{n_{k}}\right\}$ is bounded, there exists a subsequence $\left\{x_{n_{k_{l}}}\right\}$ of $\left\{x_{n_{k}}\right\}$ such that $x_{n_{k_{l}}} \rightarrow x^{*}$. Without loss of generality, we write $x_{n_{k}} \rightarrow x^{*}$. From $\omega\left(x_{n}\right) \subseteq \Omega$ so $x^{*} \in \Omega$. From $x^{+}=P_{\Omega}^{H_{1}} u$ and (5), it is deduced that

$$
\lim \sup _{n \rightarrow \infty}\left\langle u-x^{+}, x_{n}-x_{+}\right\rangle=\left\langle u-x^{+}, x^{*}-x^{+}\right\rangle \leq 0,
$$

which together with (15) and conditions (C2)-(C3), we get $\limsup _{n \rightarrow \infty} c_{n} \leq 0$. Since $\sum_{n=1}^{\infty} \alpha_{n}=\infty$ and condition (C2), we have $\sum_{n=1}^{\infty} \alpha_{n}\left(1-\beta_{n}\right)=\infty$. Hence, all conditions of Lemma 2.3 are sastified. Therefore, we immediately deduce that $s_{n} \rightarrow 0$, that is $x_{n} \rightarrow x^{+}=P_{\Omega}^{H_{1}}$. This completes the proof.

In the next method, we use the viscocity approximation one to solve a common null point problem and a variational inequality.

Theorem 3.2. If the conditions $(C 1)-(C 4)$ are satisfied, then the sequence $\left\{e_{n}\right\}$ generated by

$$
\begin{cases}u_{n} & =J_{\gamma_{n}^{A}}^{A} e_{n}, \\ v_{n} & =J_{\gamma_{n}^{B}}^{B}\left(T u_{n}\right), \\ w_{n} & =u_{n}+\delta T^{*}\left(v_{n}-T u_{n}\right), \\ e_{n+1} & =\beta_{n} e_{n}+\left(1-\beta_{n}\right)\left[\alpha_{n} f\left(e_{n}\right)+\left(1-\alpha_{n}\right) w_{n}\right], n \geq 0,\end{cases}
$$

where $f: H_{1} \rightarrow H_{1}$ is a contractive mapping from $H_{1}$ into itself with the contraction coefficient $c \in(0,1)$, converges strongly to $x^{*} \in \Omega=A^{-1} 0 \cap T^{-1}\left(B^{-1} 0\right)$ which is the unique solution of the variational inequality

$$
\left\langle(I-f) x^{*}, y-x^{*}\right\rangle \geq 0, \quad \forall x \in \Omega .
$$


Proof. Because $P_{\Omega}^{H_{1}} f$ is contractive mapping, Banach contraction mapping principle guarantees that $P_{\Omega}^{H_{1}} f$ has a unique fixed point $x^{*}$ which is also the unique solution of the variational inequality (20).

From Theorem 3.1, replacing $u$ by $f\left(x^{*}\right)$ in (5), we have the sequence $\left\{x_{n}\right\}$ converging strongly to $x^{*}=P_{\Omega}^{H_{1}} f\left(x^{*}\right)$.

Now we only need to prove that $\left\|e_{n}-x_{n}\right\| \rightarrow 0$, as $n \rightarrow \infty$. Note that

$$
\left\|e_{n+1}-x_{n+1}\right\| \leq \beta_{n}\left\|e_{n}-x_{n}\right\|+\left(1-\beta_{n}\right)\left[\alpha_{n} c\left\|e_{n}-x^{*}\right\|+\left(1-\alpha_{n}\right)\left\|w_{n}-t_{n}\right\|\right] .
$$

From Lemma 2.1 (iii), we have

$$
\begin{aligned}
\left\|w_{n}-t_{n}\right\|^{2} & =\left\|u_{n}-y_{n}\right\|^{2}+\delta^{2}\left\|T^{*}\left[\left(v_{n}-T u_{n}\right)-\left(z_{n}-T y_{n}\right)\right]\right\|^{2}+2 \delta\left\langle u_{n}-y_{n}, T^{*}\left[\left(v_{n}-T u_{n}\right)-\left(z_{n}-T y_{n}\right)\right]\right\rangle \\
& \leq\left\|u_{n}-y_{n}\right\|^{2}-\delta\left(2-\delta\|T\|^{2}\right)\left\|\left(v_{n}-T u_{n}\right)-\left(z_{n}-T y_{n}\right)\right\|^{2} \\
& \leq\left\|u_{n}-y_{n}\right\|^{2} .
\end{aligned}
$$

From the nonexpansiveness of $J_{\gamma_{n}^{A}}^{A}$, we have

$$
\left\|u_{n}-y_{n}\right\| \leq\left\|e_{n}-x_{n}\right\|
$$

Thanks to (21)-(22), we obtain

$$
\begin{aligned}
\| e_{n+1} & -x_{n+1}\left\|\leq \beta_{n}\right\| e_{n}-x_{n} \|+\left(1-\beta_{n}\right)\left[\alpha_{n} c\left\|e_{n}-x^{*}\right\|+\left(1-\alpha_{n}\right)\left\|e_{n}-x_{n}\right\|\right] \\
& \left.\leq\left[1-\alpha_{n}\left(1-\beta_{n}\right)\right]\left\|e_{n}-x_{n}\right\|+\alpha_{n}\left(1-\beta_{n}\right) c\left(\left\|e_{n}-x_{n}\right\|+\left\|x_{n}-x^{*}\right\|\right]\right) \\
& \left.=\left[1-\alpha_{n}\left(1-\beta_{n}\right)(1-c)\right]\left\|e_{n}-x_{n}\right\|+\alpha_{n}\left(1-\beta_{n}\right) c\left(\left\|x_{n}-x^{*}\right\|\right]\right) .
\end{aligned}
$$

From Lemma 2.3, we get $\lim _{n \rightarrow \infty}\left\|e_{n}-x_{n}\right\|=0$. Thus, $\lim _{n \rightarrow \infty}\left\|e_{n}-x^{*}\right\|=0$, we obtain that $\left\{e_{n}\right\}$ generated by (19) converges strongly to $x^{*}=P_{\Omega}^{H_{1}} f\left(x^{*}\right)$.

\section{Numerical Results}

In the following example, for the convergence illustration of iterative methods (5) and (19) studied in Theorems 3.1 and 3.2, respectively, we present a numerical example which is finding a common solution of a common null point problem and a variational inequality in Euclidean spaces. We perform the iterative schemes in MATLAB R2016a running on a laptop with Intel(R) Core(TM) i3-5200U CPU @ 2.20 GHz, RAM 10 GB.

Let $H_{1}=\mathbb{R}^{2}, H_{2}=\mathbb{R}^{5}$. Mapping $f: \mathbb{R}^{2} \rightarrow \mathbb{R}^{2}$, where $f(x)=\frac{1}{2} x$ is a contractive mapping with the contration coefficient $c=\frac{1}{2}$. In $\mathbb{R}^{2}$, the maximal monotone operator $A$ is defined as

$$
A\left(x_{1}, x_{2}\right)=\left(2 x_{1}+2 x_{2}, 2 x_{1}+2 x_{2}\right)^{T}
$$

and in $H_{2}$, the maximal monotone operator $B$ is defined as

$$
B x=\left(\begin{array}{ccccc}
0 & 0 & 0 & 0 & 0 \\
0 & 1 & 1 & 0 & 0 \\
0 & -1 & 1 & 0 & 0 \\
0 & 0 & 0 & 1 & 1 \\
0 & 0 & 0 & 0 & 1
\end{array}\right) x
$$

where $x=\left(x_{1}, x_{2}, x_{3}, x_{4}, x_{5}\right) \in \mathbb{R}^{5}$. Let $T: \mathbb{R}^{2} \rightarrow \mathbb{R}^{5}$ be a bounded linear operator defined by

$$
T\left(x_{1}, x_{2}\right)=\left(x_{1}+x_{2}, 2 x_{1}, 3 x_{1}+4 x_{2}, 0, x_{1}+x_{2}\right)^{T},
$$

such that $\Omega=A^{-1} 0 \cap T^{-1}\left(B^{-1} 0\right) \neq \emptyset$.

We will use method (5) to solve the null point problem which is finding a $x^{+} \in \Omega$ such that $x^{+}=P_{\Omega}^{H_{1}} u$ for any $u, x_{0} \in H_{1}$, knowing that the exact solution of the considered problem is $x^{*}=(0,0) \in \mathbb{R}^{2}$. The following table shows the approximate solutions $x_{n}=\left(x_{n}^{1}, x_{n}^{2}\right) \in \mathbb{R}^{2}$ of the above problem with the corresponding parameters. Next, we find $x^{+} \in \Omega$ that is also the solution of the variational inequality $\left\langle(I-f) x^{*}, y-x^{*}\right\rangle \geq 0, \forall y \in \Omega$. Using (19), we have the approximate solution shown in following table. 


\begin{tabular}{cccccc}
\hline $\operatorname{Iter}(n)$ & $x_{n}^{1}$ & $x_{n}^{2}$ & $\operatorname{Iter}(n)$ & $x_{n}^{1}$ & $x_{n}^{2}$ \\
\hline \hline 1 & -2.0000 & 1.0000 & 50 & -0.052869 & 0.039805 \\
2 & -1.4022 & 0.79506 & 100 & -0.0066537 & 0.0049955 \\
3 & -1.0927 & 0.68717 & 300 & $-6.6336 \mathrm{e}-06$ & $-1.2403 \mathrm{e}-06$ \\
10 & -0.45866 & 0.34394 & 500 & $-1.7653 \mathrm{e}-06$ & $-2.4079 \mathrm{e}-06$ \\
\hline
\end{tabular}

Table 1: $x_{0}=(-2,1)^{T}, u=(-1 / 100,-1 / 100)^{T}, \delta=0.05, \gamma_{n}^{A}=\gamma_{n}^{B}=1, \beta_{n}=\frac{1}{2}, \alpha_{n}=\frac{1}{n+1}$

\begin{tabular}{cccccc}
\hline Iter $(n)$ & $x_{n+1}^{1}$ & $x_{n+1}^{2}$ & $\operatorname{Iter}(n)$ & $x_{n+1}^{1}$ & $x_{n+1}^{2}$ \\
\hline \hline 1 & -2.0000 & 1.0000 & 50 & -0.13956 & 0.10517 \\
2 & -1.6519 & 0.92031 & 100 & -0.020987 & 0.015816 \\
3 & -1.4221 & 0.87588 & 300 & $-1.5558 \mathrm{e}-05$ & $1.1725 \mathrm{e}-05$ \\
10 & -0.80129 & 0.6003 & 500 & $-1.3146 \mathrm{e}-08$ & $9.9068 \mathrm{e}-09$ \\
\hline
\end{tabular}

Table 2: $x_{0}=(-2,1)^{T}, \delta=0.05, \gamma_{n}^{A}=\gamma_{n}^{B}=1, \beta_{n}=\frac{1}{2}, \alpha_{n}=\frac{1}{n+1}$

\section{Conclusion}

We have presented in this paper the two iterative methods based on Halpern method and the viscosity one to solve a common null point problem and a variational inequality in Hilbert spaces. The strong convergence of the methods is proven under some certain assumptions and a numerical example for the convergence illustration of the proposed method is given.

\section{Acknowledgments}

The two authors would like to thank the refrees for their useful suggestions and comments that help to improve the presentation of this paper.

\section{REFERENCES}

[1] Y. Censor and T. Elfving, "A multi projection algorithm using Bregman projections in a product space," Number. Algo., vol. 8, no. 2-4, pp. 221-239, 1994.

[2] Y. Censor, T. Elfving, N. Kopf, and T. Bortfeld, "The multiple-sets split feasibility problem and its application," Inverse Problems, vol. 21, pp. 2071-2084, 2005.

[3] Y. Censor, T. Bortfeld, B. Martin, and A. Trofimov, "A unified approach for inversion problems in intensitymodulated radiation therapy," Phys. Med. Biol., vol. 51, pp. 2353-2365, 2006.

[4] T. M. Tuyen, N.T. T. Thuy, and N. M. Trang, "Strong convergence theorems of a split common null point problem and a fixed point problem in Hilbert Spaces," Appl. Set-Valued Anal. Optim., vol. 2, pp. 205-222, 2020.

[5] G. Stampacchia, "Formes bilineaires coercivites sur les ensembles convexes," Comptes Rendus de l'Académie des Sciences, vol. 258, pp. 4413-4416, 1964.

[6] V. Barbu and T. Precupanu, Convexity and optimization in Banach spaces, Springer, Netherlands, 2012.

[7] E. Bonacker, A.Gibali, and K.H. Kufer, "Nesterov perturbations and projection methods applied to IMRT," J. Nonlinear Var. Anal., vol. 4, pp. 63-86, 2019.

[8] T. M. Tuyen, "A strong convergence theorem for the split common null point problem in Banach spaces," Appl. Math. Optim., vol. 79, pp. 207-227, 2019.

[9] B. Halpern, "Fixed points of nonexpansive maps," Bull. Amer. Math. Soc., vol. 73, pp. 591-597, 1967.

[10] T. Suzuki, "Strong convergence theorems for infinite families of nonexpansive mappings in general Banach spaces," Fixed Point Theory Appl., vol. 1, pp. 103-123, 2005. 